\title{
RELACIÓN ENTRE ANTI-INFLAMATORIOS NO ESTEROIDEOS (AINES) Y QUERATOLISIS. A PROPÓSITO DE UN CASO
}

\section{CORNEAL MELTING AND TOPICAL NONSTEROIDAL ANTI- INFLAMMATORY DRUG (NSAID). A CASE REPORT}

\author{
MONTES-MOLLÓN MA ${ }^{1}$, PÉREZ-RICO C ${ }^{2}$, BECKFORD-TÖNGREN C ${ }^{1}$, CASTRO Mำ, \\ PAREJA-ESTEBAN J ${ }^{1}$, ROMERO-GARCÍA A ${ }^{2}$
}

\begin{abstract}
RESUMEN
Objetivo: Establecer la posible relación entre el uso de los antiinflamatorios no esteroideos (AINEs) tópicos y la queratolisis.

Caso clínico: Se presenta un caso de queratolisis tras cirugía vitreorretiniana y uso tópico de ketorolaco trometamol (Acular ${ }^{\circledR}$ ).

Discusión: Se plantea la etiología multifactorial en los casos de queratolisis asociados al uso de AINEs y recomendando un uso de los mismos adecuado a cada situación, valorando la posible comorbilidad de otros agentes.

Palabras clave: Ketorolaco, queratolisis, AINEs, cirugía vitreorretiniana, enfermedad de superficie ocular.
\end{abstract}

\begin{abstract}
Purpose: To study the relationship between the use of nonsteroidal anti-inflammatory drug (NSAID) and the corneal melting.

Case report: We report a case of keratolysis after vitreoretinal surgery and topical use of ketorolac tromethamine (Acular $\left.{ }^{\circledR}\right)$.

Discussion: It is proposed the multifactorial etiology in the cases of corneal melting related to the use of NSAID and it is recommended an reasonable use of them in each situation and to check the possible comorbility of other agents (Arch Soc Esp Oftalmol 2009; 84: 311-314).
\end{abstract}

Key words: Ketorolac, corneal melting, NSAID, vitreoretinal surgery, ocular surface desease.

\section{INTRODUCCIÓN}

Actualmente los anti-inflamatorios no esteroideos (AINEs) tópicos se utilizan de forma frecuente en el postoperatorio de diversas cirugías oculares para controlar el dolor y la inflamación.

Son pocas las complicaciones relacionadas con su uso, aunque se han descrito: queratitis puntata, infiltrados subepiteliales, infiltrados estromales, anillos inmunes, defectos corneales persistentes y en casos graves queratolisis $(1,2)$.

En 1999 la Sociedad Americana de Cirugía de Catarata y Refractiva (ASCRS), llevó a cabo una revisión de las complicaciones corneales asociadas al uso de AINEs. Si bien los primeros casos publicados se atribuían al diclofenaco sódico genérico (Falcon, Alcon Laboratorios, Ft Worth, TX), también se han descrito casos relacionados con diclofe-

\footnotetext{
Recibido: 29/5/07. Aceptado: 23/6/09.

Servicio de Oftalmología. Hospital Universitario Príncipe de Asturias de Alcalá. España.

1 Licenciado en Medicina.

2 Doctor en Medicina.

Correspondencia:

M. a Ángeles Montes Mollón

C/. Padre Claret, n. ${ }^{\circ} 7$ B

28002 Madrid

España

E-mail: mmontesv28@gmail.com
} 
naco sódico de marca registrada (Voltaren ${ }^{\circledR}$, Ciba Vision, Atlanta, GA) y ketorolaco trometamina (Acular ${ }^{\circledR}$, Allergan Inc, Irving, CA), tanto si son prescritos en forma de colirio como en monodosis (libres de preservantes) (1). Más recientemente ha sido aprobado en Japón el uso tópico de bromfenaco sódico, un nuevo AINE, que también se ha asociado a graves complicaciones corneales (3).

Presentamos a continuación un caso de queratolisis asociado al uso de colirio de ketorolaco trometamol $\left(\right.$ Acular $^{\circledR}$, Allergan Inc, Madrid) tras cirugía vitreorretiniana. Se discute la posible etiología multifactorial de este tipo de complicación y las recomendaciones para la adecuada prescripción de los AINEs.

\section{CASO CLÍNICO}

Paciente de 70 años de edad, sin antecedentes sistémicos de interés, es intervenido de catarata con luxación posterior del núcleo cristaliniano. Se instauró tratamiento con Tobradex ${ }^{\circledR}$ (Alcon Cusí Inc, Barcelona) (1 gota $\mathrm{x} 4$ veces al día), Timoftol $0,5 \%{ }^{\circledR}$ (Merk Sharp \& Dome Inc, Madrid) (1 gota $\mathrm{x}$ 2 veces al día) y Acular (1 gota x 4 veces al día). A los 15 días se realiza vitrectomía con facofragmentación del núcleo e implante de lente intraocular en cámara anterior y se mantiene tratamiento con Acular y Tobradex. A los 10 días el paciente presenta dolor intenso, enrojecimiento, lagrimeo y blefaroespasmo. A la exploración biomicroscópica se observa inyección ciliar intensa, adelgazamiento corneal periférico con defecto epitelial de $5 \times 3 \mathrm{~mm}$ e infil-

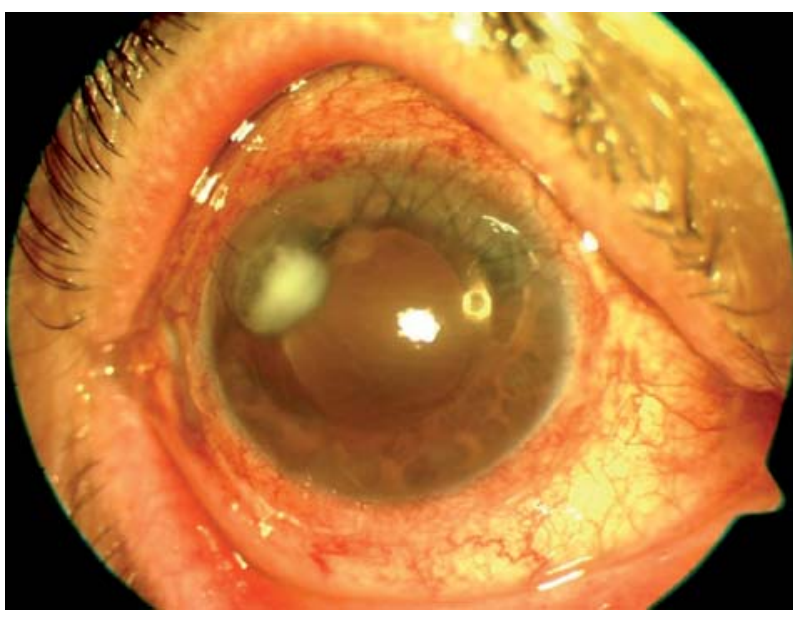

Fig. 1: Adelgazamiento corneal periférico con infiltración estromal. tración estromal perilesional (fig. 1). Se realiza cultivo y se instaura terapia intensiva con Ciprofloxacino $^{\circledR}$ (Farma-Lepori Inc, Barcelona) manteniéndose el Acular. El cultivo fue negativo y al no objetivarse mejoría, se suspende el tratamiento y se pauta tratamiento con Medrivas ${ }^{\circledR}$ (Alcon Cusi Inc, Barcelona), lágrimas artificiales, colirio de Aureomicina $^{\circledR}$ (Alcon Cusi Inc, Barcelona) y Redoxón ${ }^{\circledR}$ (Bayer Inc, ). A la semana desaparece la infiltración y se restablece el grosor normal de la córnea quedando una leve opacidad corneal.

\section{DISCUSIÓN}

Los AINES son fármacos anti-inflamatorios que inhíben la actividad de las ciclooxigenasas (COXs), disminuyendo la síntesis de prostaglandinas y reduciendo el dolor y la inflamación postoperatoria.

Mucho se ha hablado de la relación entre el uso de los AINEs y la queratolisis. El mecanismo por el que los AINES producen queratolisis no es del todo conocido. Posibles factores son: inhibición de la proliferación de queratocitos, alteraciones en la regulación y expresión de las metaloproteinasas de la matriz extracelular (MMP-1, MMP-2 y MMP-8) y disminución de la sensibilidad corneal, con retraso en la cicatrización corneal (2). Además, altas dosis de AINEs pueden estimular la inflamación, ya que aunque normalmente inhíben las COXs, altas concentraciones inducen mRNAs y proteínas de COX1 y COX2. Por otra parte la inhibición de las COXs puede potenciar la vía de la lipooxigenasa y sus derivados como los leucotrienos, aumentando la respuesta celular inflamatoria (2).

¿Quiere decirse que los AINEs son peligrosos y requieren precaución en su uso? No está del todo demostrado. En los casos registrados, se observan mecanismos multifactoriales como causantes de queratolisis. Así, superficies oculares comprometidas, asociaciones con otros fármacos, posologías indebidas y prolongadas, uso de lentes de contacto e indicaciones no correctas pueden haber actuado individualmente o en asociación como causa de queratolisis (2-4).

Nuestro caso tenía como factor ocular predisponente las dos cirugías en corto espacio de tiempo, la asociación con Tobradex y Ciprofloxacino junto con sus preservantes.

Así pues, pacientes con patología de superficie ocular: queratoconjuntivitis sicca, deficiencia limbar, queratitis neurotrófica, de exposición, defectos epiteliales persistentes o enfermedades del coláge- 
no se considerarían contraindicaciones relativas al uso de AINEs. Y si se utilizaran sería conveniente un seguimiento muy frecuente para evitar posibles complicaciones corneales.

En cuanto a la posología y duración del tratamiento en cirugía refractiva de superficie se recomiendan durante un corto periodo de tiempo (2 ó 3 días) 4 veces al día, aunque hay autores que los mantienen entre 2 y 3 semanas para modular la hipercorrección quirúrgica (5), y tras cirugía de catarata la dosis es de dos semanas 4 veces al día para el diclofenaco sódico y el ketorolaco y si se utiliza el bromfenaco sódico han de ser 2 veces al día.

Concluimos que siempre que se prescriban los AINEs es conveniente descartar posibles factores que puedan contribuir a toxicidad corneal.

\section{BIBLIOGRAFÍA}

1. Congdon NG, Schein OD, von Kulajta P. Corneal complications associated with topical ophthalmic use of nonsteroidal anti.inflammatory drugs. J Cataract Refract Surg 2001; 27: 622-631.

2. Mian SI, Gupta A, Pineda R. Corneal ulceration and perforation with ketorolac tromethamine $\left(\right.$ Acular $\left.^{\circledR}\right)$ use after PRK. Cornea 2006; 25: 232-234.

3. Asai T, Nakagami T, Mochizuki M, Hata N, Tsuchiya T, Hotta Y. Three cases of corneal melting after instillation of a new nonsteroidal anti-inflammatory drug. Cornea 2006; 25: 224-227.

4. Zanini M, Savini G, Barboni P. Corneal melting associated with topical diclofenanac use after laser-assisted subepithelial keratectomy. J Cataract Refract Surg 2006; 32: 1570-1572.

5. Yee RW, Yee SB. Update on laser subepithelial keratectomy (LASEK). Curr Opin Ophthalmol 2004; 15: 333-341. 
